\title{
DIFFERENTIABILITY OF CONTINUOUS HOMOMORPHISMS BETWEEN SMOOTH LOOPS
}

\author{
by
}

Richard Bödi and Linus Kramer

\begin{abstract}
It is a well-known fact that a continuous homomorphism between Lie groups is analytic. We prove a similar result (Thm. 1.8) for continuous homomorphisms of differentiable left or right loops in section 1 of this paper. Section 2 deals with images and kernels of such homomorphisms. Again, the results obtained are quite analogous to the Lie group case. The paper ends with applications of Theorem 1.8. For example, it turns out that the group of continuous automorphisms of a smooth generalized polygon is a Lie transformation group with respect to the compact-open topology.
\end{abstract}

AMS-Subject Classification : 12K99, 20N05, 57N45

Keywords : loops, morphisms, continuity, differentiability, smoothness.

Throughout this paper, we assume all objects to be smooth of class $\mathbf{C}^{\mathbf{k}}$ for some fixed integer $k$ with $2 \leq k \leq \infty$, unless stated otherwise explicitly.

\section{Continuous homomorphisms of smooth loops}

First, we need the algebraic notion of a loop:

(1.1) Definition. A left loop $(K, e, 0)$ consists of a set $K$, an element $e \in K$, called the neutral element, and a map $\circ: K \times K \rightarrow K$ with the following two properties: $x \circ e=e \circ x=x$ for all elements $x \in K$, and the equation $a \circ x=b$ has a unique solution $x$ for every pair $a, b \in K$. We put $x=a \backslash b$. This yields the identity $x \circ(x \backslash a)=a$. Substituting $x \circ a$ for $a$, we find $x \backslash(x \circ a)=a$.

Similarly, we define a right loop; in this case, we require that $x \circ a=b$ has a unique solution $b / a$. If both equations have unique solutions, then $(K, e, 0)$ is simply called a loop.

(1.2) Definition. An $n$-dimensional smooth local $H$-space $\mathcal{K}=(K, U, e, o)$ consists of a smooth $n$-manifold $K$, an open subset $U$ of $K$ with $e \in U$ and a smooth map o: $U \times U \rightarrow K$ with the following property:

$$
x \circ e=e \circ x=x \text { for every element } x \in U .
$$


The left translation $x \mapsto \lambda_{a} x$ is defined by $\lambda_{a} x=a \circ x$.

A left loop $(K, e, 0)$ is called an $n$-dimensional smooth left loop if $K$ is a smooth $n$ manifold, and if the maps $o$ and $\backslash$ are smooth. Similarly, we define a smooth right loop and a smooth loop. For every $a \in K$, the left translation map $\lambda_{a}$ is a diffeomorphism with inverse $x \mapsto \lambda_{a}^{-1} x=a \backslash x$.

The next proposition shows that every smooth local H-space is a smooth (left and right) loop near the neutral element, i.e. it is a local loop in the sense of Kozma [7] and in the sense of Hofmann and Strambach [6].

(1.3) Proposition. Let $\mathcal{K}=(K, U, e, 0)$ be a smooth local $\mathrm{H}$-space. Then there exists a smooth map $(x, y) \mapsto x \backslash y$ defined on a neighborhood $V$ of $(e, e)$, such that $x \circ(x \backslash a)=a$, whenever the left hand side is defined. Hence the left translation $\lambda_{a}$ is a diffeomorphism for all $a$ in some neigborhhood $V \subseteq U$ of $e$.

Proof. Consider the smooth map $f:(x, y) \mapsto(x, x \circ y): U \times U \rightarrow K \times K$. Because of the relation $x \circ e=e \circ x=x$, its derivative at $(e, e)$ is given by $\mathrm{D} f_{(e, e)}=\left(\begin{array}{ll}1 & 0 \\ 1 & 1\end{array}\right)$. Hence $f$ has a smooth local inverse $f^{-1}:(x, a) \mapsto(x, y)=(x, x \backslash a)$ near $(e, e)$.

In our studies the notion of a canonical coordinate system in the sense of [1] and [7] plays a dominant rôle.

(1.4) Definition. Let $(K, U, e, 0)$ be an $n$-dimensional smooth local $\mathrm{H}$-space, and let $V$ be a neighborhood of $e$. A coordinate chart $h:(V, e) \rightarrow\left(\mathbb{R}^{n}, 0\right)$ is called a canonical coordinate system, if the relation

$$
h(x \circ x)=h(x)+h(x)
$$

holds for every element $x$ with $x \circ x \in V \cap U$. Note that for every $H \in \mathbf{G L}_{n} \mathbb{R}$ the composite $H h$ is again a canonical coordinate system, and conversely every other canonical coordinate system arises in this way, cp. Kozma [7] and Theorem (1.8).

A surprising result of J. Kozma [7] states that there exists a canonical coordinate system for every smooth local H-space. Note that in [7] this theorem is formulated for smooth local loops rather than for H-spaces, although J. Kozma's proof does not use the fact that the loop operation has local inverses. The proof for the existence of a canonical coordinate system is based on a variation of a theorem of Sternberg [13], Thm. 2 on contractions, which is formulated below. A proof of it can be found in the appendix of $[7]$. 
(1.5) Theorem. Let $M$ be a smooth $n$-manifold and let $f: M \rightarrow M$ be a smooth function that fixes an element $e \in M$. If the derivative $\mathrm{D} f_{e}$ at $e$ satisfies $\mathrm{D} f_{e}=\frac{1}{2} \cdot \mathbb{1}$, then there exists an open neighborhood $U$ of $e$ and a coordinate chart $h:(U, e) \rightarrow\left(\mathbb{R}^{n}, 0\right)$ such that $h(f(x))=\frac{1}{2} h(x)$ for every $x \in U$.

Using J. Kozma's version of the theorem of Sternberg it is easy to prove the following result, which can also be found in [7].

(1.6) Theorem. For every smooth local $\mathrm{H}$-space $(K, U, e, o)$ there exists a canonical coordinate system $h$.

Proof. Consider the smooth map Sq: $x \mapsto x \circ x: U \rightarrow K$. Using the relation $x \circ e=e \circ x=x$, it is readily verified that its derivative at $e$ is $\mathrm{DSq}_{e}=2 \cdot \mathbb{1}$. Thus $\mathrm{Sq}$ has a local inverse Sqrt in some neighborhood of $e$, and DSqrt $e=\frac{1}{2} \cdot \mathbb{1}$. So we may apply Sternberg's theorem to get a coordinate chart $h:(V, e) \rightarrow\left(\mathbb{R}^{n}, 0\right)$ with $h(\operatorname{Sqrt}(x))=\frac{1}{2} h(x)$, and this of course implies that $h(x \circ x)=h(x)+h(x)$.

(1.7) Definition. A continuous local homomorphism between two local H-spaces $\mathcal{K}=$ $(K, U, e, o)$ and $\mathcal{K}^{\prime}=\left(K^{\prime}, U^{\prime}, e^{\prime}, o^{\prime}\right)$ is a continuous map $f:(V, e) \rightarrow\left(K^{\prime}, e^{\prime}\right)$ defined on some neighborhood $V$ of $e$ such that the relation

$$
f(x \circ y)=f(x) \circ^{\prime} f(y)
$$

is satisfied whenever both sides of the equation are defined. A continuous homomorphism between two (left, right) loops is a local homomorphism that is defined on all of $K$.

A smooth local (global) homomorphism $f$ between $\mathcal{K}$ and $\mathcal{K}^{\prime}$ is a continuous local (global) homomorphism which is a smooth map.

The following is our first main result. It generalizes a well-known theorem about Lie groups, see Warner [14], Thm.3.39, p. 109 or Hochschild [5], Chapt. VII, Thm.4.2, e.g. For Lie groups the standard proof works with local one-parameter subgroups which need not exist in smooth local $\mathrm{H}$-spaces, and it uses properties of the exponential mapping which our canonical coordinates do not have in general. Hence our approach is completely different. The main idea is to show that a continuous homomorphism between smooth local $\mathrm{H}$-spaces is Lipschitz-continuous and hence it possesses points of differentiability almost everywhere.

(1.8) Theorem. Every continuous local homomorphism $f: \mathcal{K} \rightarrow \mathcal{X}^{\prime}$ between smooth local H-spaces $\mathcal{K}$ and $\mathcal{K}^{\prime}$ is smooth in a neighborhood of the neutral element $e$. In fact, $f$ is locally a linear map with respect to canonical coordinate systems on $\mathcal{K}$ and $\mathcal{K}^{\prime}$, respectively. 
Proof. Passing to canonical coordinates, we may assume that $(K, e)=\left(\mathbb{R}^{n}, 0\right),\left(K^{\prime}, e^{\prime}\right)=$ $\left(\mathbb{R}^{n^{\prime}}, 0\right)$. Choosing suitable euclidean metrics on $\mathbb{R}^{n}$ and $\mathbb{R}^{n^{\prime}}$, we may also assume that $f$ maps the unit ball $B \subseteq \mathbb{R}^{n}$ into the unit ball $B^{\prime} \subseteq \mathbb{R}^{n^{\prime}}$, and that the maps o, $\backslash, o^{\prime}$, and $\backslash^{\prime}$ are defined on $B \times B$ and $B^{\prime} \times B^{\prime}$, respectively. We put $\mathrm{Sq}(x)=x \circ x$, Sqrt $=\mathrm{Sq}^{-1}$ and $\mathrm{Sqrt}^{\prime}=\left(x^{\prime} \mapsto x^{\prime} \circ^{\prime} x^{\prime}\right)^{-1}$ as in the proof of (1.3). We may also assume that these maps are defined on $B$ and $B^{\prime}$, repectively.

We want to show first that the continuous homomorphism $f$ satisfies a Lipschitz condition in some neighborhood of the origin. Since $o^{\prime}$ and $\backslash$ are smooth, we can find constants $C_{1}, C_{2}$ such that

$$
\left|a^{\prime} \circ^{\prime} b^{\prime}-x^{\prime} \circ^{\prime} y^{\prime}\right| \leq C_{1}\left(\left|a^{\prime}-x^{\prime}\right|+\left|b^{\prime}-y^{\prime}\right|\right)
$$

and

$$
|a \backslash b-x \backslash y| \leq C_{2}(|a-x|+|b-y|)
$$

on $B^{\prime} \times B^{\prime}$ and $B \times B$, respectively. Since $\mathrm{Sq}^{k}(x)=2^{k} x$ if $2^{k-1} x \in B$, we can find for every $x \in B \backslash\{0\}$ an integer $m$ such that $\frac{1}{2} \leq\left|\mathrm{Sq}^{m} x\right|<1$. This of course implies that $2^{-m} \leq 2|x|$. Now

$$
\begin{aligned}
|f(x)| & =\left|f\left(\operatorname{Sqrt}^{m}\left(\operatorname{Sq}^{m}(x)\right)\right)\right|=\left|\operatorname{Sqrt}^{\prime m}\left(f\left(\mathrm{Sq}^{m}(x)\right)\right)\right| \\
& =2^{-m}\left|f\left(\operatorname{Sq}^{m}(x)\right)\right| \leq 2^{-m} \leq 2|x|,
\end{aligned}
$$

because $f(B) \subseteq B^{\prime}$. Choose a neighborhood $U$ of 0 with $U \circ U \subseteq B$. For $x, y \in U$ we get

$$
\begin{aligned}
|f(x \circ y)-f(x)| & =\left|f(x) \circ^{\prime} f(y)-f(x) \circ^{\prime} f(0)\right| \leq C_{1}|f(y)-f(0)| \\
\leq 2 C_{1}|y| & =2 C_{1}|x \backslash(x \circ y)-x \backslash x| \leq 2 C_{1} C_{2}|x \circ y-x|,
\end{aligned}
$$

and therefore, the homomorphism $f$ satisfies a Lipschitz condition on $U$.

Applying Stein [12], Thm. 3, p. 250 to the $n^{\prime}$ coordinate functions of $f$, we infer that the map $f$ is differentiable almost everywhere in $U$. Let $x_{0} \in U$ be such a point. Choose an arbitrary point $x_{1} \in U$ and let $a \in B$ such that $\lambda_{a}\left(x_{0}\right)=x_{1}$. Since we may write

$$
f=f \lambda_{a} \lambda_{a}^{-1}=\lambda_{f(a)}^{\prime} f \lambda_{a}^{-1},
$$

it follows that $f$ is also differentiable at $x_{1}$. Thus the map $f$ is differentiable on $U$. By [8], the homomorphism $f$ is locally a linear map and consequently is smooth.

(1.9) Theorem. Every continuous homomorphism $f: \mathcal{X} \rightarrow \mathcal{X}^{\prime}$ between smooth (left, right) loops $\mathcal{X}$ and $\mathcal{K}^{\prime}$ is smooth. In particular, the differentiable structure of a smooth loop is uniquely determined by the underlying topological loop.

Proof. Choose an arbitrary element $a \in K$. Since $f=\lambda_{f(a)}^{\prime} f \lambda_{a}^{-1}$ on $K$ and since $f$ is a smooth mapping in a neighborhood of the neutral element $e$ by (1.8), it follows that $f$ is smooth in a neighborhood of $a$. 


\section{Images and kernels}

(2.1) Theorem. Let $f: \mathcal{X} \rightarrow \mathcal{X}^{\prime}$ be a continuous homomorphism between smooth (left, right) loops. Then $f$ is a smooth map of constant rank, and therefore the kernel $N=f^{-1}\left(e^{\prime}\right)$ is a smoothly imbedded submanifold of codimension rank $f$ in $K$, and a smooth (left, right) loop with respect to the induced differentiable structure.

Proof. The map $f$ is smooth by (1.9). Let $a \in K$. Now $f=\lambda_{f(a)}^{\prime} f \lambda_{a}^{-1}$, hence $\mathrm{D} f_{a}=$ $\left(\mathrm{D} \lambda_{f(a)}^{\prime}\right)_{e} \mathrm{D} f_{e}\left(\mathrm{D} \lambda_{a}^{-1}\right)_{a}$. Thus the derivative of $f$ has constant rank on $K$, and therefore the fibers of $f$ are smoothly imbedded submanifolds, cp. Spivak [11] Ch.2, Prop. 12.

The next theorem generalizes the topological situation (see [6], IX.1.9) to the smooth case.

(2.2) Theorem. Let $f: \mathcal{X} \rightarrow \mathcal{X}^{\prime}$ be a continuous homomorphism between smooth (left, right) loops with kernel $N$. Let $K / N$ denote the quotient space obtained from collapsing the fibers of $f$. Then $K / N$ has a unique differentiable structure such that it becomes a smooth (left, right) loop, and such that the projection map $\pi: K \rightarrow K / N$ is a smooth homomorphism. Let $\tilde{f}$ denote the induced map $\tilde{f}: K / N \rightarrow K^{\prime}$. Then $\tilde{f}$ is an injective immersion and a smooth homomorphism, and $f$ factors as $f=\tilde{f} \pi$.

We divide the proof into three steps.

(1) The fiber of $f$ over $f(a)$ is exactly the 'coset' $\lambda_{a} N=a \circ N$. The quotient space $K / N$ obtained by collapsing the fibers is a second countable, locally euclidean Hausdorff space, and the projection $\pi: K \mapsto K / N$ is a locally trivial fibration.

Let $a, b$ be elements of $K$. Then $f(a)=f(b)$ if and only if $a \backslash b \in N$, and this is equivalent to $b \in a \circ N$. Thus the fibers of $f$ are exactly the left translates of $N$. Since $\tilde{f}: K / N \rightarrow K^{\prime}$ is a continuous injection into a Hausdorff space, $K / N$ is a Hausdorff space as well.

Let $k$ denote the codimension of the submanifold $N \subseteq K$. Since $f$ has constant rank $k$, we can find an imbedded submanifold $S$ diffeomorphic to $\mathbb{R}^{k}$ in $K$ containing $e$ and transversal to the fibers of $f$, such that the restriction $\left.f\right|_{S}$ is a diffeomorphism onto $f(S) \subseteq K^{\prime}$, and such that the map $\xi: a \mapsto(a \circ N) \cap S$ is smooth near $S$, cp. Spivak [11] Ch.2, Thm. 9(2). For $a \in K$, we put $S_{a}=\lambda_{a}(S)$. We claim that the map $(s, x) \mapsto s \circ x$ is a homeomorphism between $S_{a} \times N$ and $S_{a} \circ N=\pi^{-1} \pi\left(S_{a}\right)$. Indeed, if $(a \circ s) \circ x=\left(a \circ s^{\prime}\right) \circ x^{\prime}$, then $f(a) \circ^{\prime} f(s)=f(a) \circ^{\prime} f\left(s^{\prime}\right)$, and thus $s=s^{\prime}$ and $x=x^{\prime}$. By invariance of domain, the map is a homeomorphism. Note that the restriction $\pi_{a}=\left.\pi\right|_{S_{a}}$ is a homeomorphism between $S_{a}$ and the image $\tilde{S}_{a}=\pi\left(S_{a}\right)$. This shows that $\pi$ is a locally trivial fibration, and hence an open surjection.

(2) Consider the collection of homeomorphisms $\mathfrak{A}=\left\{\left(\pi_{a} \lambda_{a}\right)^{-1}: \tilde{S}_{a} \rightarrow S \cong \mathbb{R}^{k} \mid a \in K\right\}$. We claim that this collection is a smooth atlas for $K / N$. This implies that $\pi$ is a smooth map of rank $k$, and that the maps $\pi_{a}$ are diffeomorphisms. 
First note that $f=\lambda_{f(a)}^{\prime} f \lambda_{a}^{-1}$, hence the restriction $\left.f\right|_{S_{a}}: S_{a} \rightarrow f\left(S_{a}\right)$ is a diffeomorphism. Consider the composite $\varphi=\left(\lambda_{a}^{-1} \pi_{a}^{-1}\right)\left(\pi_{b} \lambda_{b}\right)$. Now $\pi_{a}^{-1} \pi_{b}=\left(\left.f\right|_{S_{a}}\right)^{-1}\left(\left.f\right|_{S_{b}}\right)$ is smooth, hence $\varphi$ is smooth wherever it is defined. Thus the collection $\mathfrak{A}$ defines a differentiable structure on $K / N$. The maps $\pi_{a}$ are diffeomorphisms by definition. The fact that $\pi$ is smooth near $a \in K$ follows from the identity $\left(\pi_{a} \lambda_{a}\right)^{-1} \pi \lambda_{a}=\xi$.

(3) Since $K / N \rightarrow f(K)$ is a bijection, the quotient space becomes a (left, right) loop with respect to the maps $(a \circ N) \tilde{o}(b \circ N)=(a \circ b) \circ N$ and $(a \circ N) \tilde{\Upsilon}(b \circ N)=(a \backslash b) \circ N$. We claim that $K / N$ is in fact a smooth (left, right) loop.

Let $a \circ N$ and $b \circ N$ be elements of $K / N$. For elements $x$ and $y$ close to $a \circ N$ and $b \circ N$, respectively, we may write $x$ õ $y=\pi\left(\pi_{a}^{-1}(x) \circ \pi_{b}^{-1}(y)\right)$ and $x \tilde{\backslash} y=\pi\left(\pi_{a}^{-1}(x) \backslash \pi_{b}^{-1}(y)\right)$, hence the maps $\tilde{o}$ and $\tilde{\nearrow}$ are smooth. Finally, $\tilde{f}$ is continuous and hence a smooth immersion.

\section{Applications}

Using [2], (2.10) we get the following result as an immediate corollary from (1.9).

(3.1) Corollary. For every connected smooth double loop $\mathcal{D}$ in the sense of [2], (2.5) the group Aut $(\mathcal{D})$ of continuous automorphisms of $\mathcal{D}$ coincides with the group of smooth automorphisms of $\mathcal{D}$, and $\operatorname{Aut}(\mathcal{D})$ is a compact linear Lie group with respect to the compact-open topology.

As a further application we shall prove the following result in a forthcoming paper.

(3.2) Theorem. Every continuous collineation between two smooth generalized polygons is smooth. In particular, every continuous collineation of a smooth projective plane is smooth.

Consequences of this theorem are the following corollaries.

(3.3) Corollary. Every compact generalized polygon possesses at most one differentiable structure such that it becomes a smooth generalized polygon. Moreover, every punctured point row and every punctured pencil of lines is diffeomorphic to some euclidean space.

From Salzmann [10], §2 or Grundhöfer [4], Thm. 1, Burns-Spatzier [3], 2.1, and Montgomery-Zippin [9], Ch. V, we moreover obtain

(3.4) Corollary. The group of continuous automorphisms of a smooth generalized polygon is a smooth Lie transformation group with respect to the compact-open topology. 


\section{References}

[1] Akivis, M.A., Canonical expansions of the equation of a local analytic quasigroup, Sov. Math. Dokl. 10 (1969), 1200-1203

[2] Bödi, R., Automorphism Groups of Differentiable Double Loops, Geometriae Dedicata 46 (1993), 61-72

[3] Burns, K., Spatzier, R., On topological Tits buildings and their classification, Publ. Math. IHES 65 (1987), 5-34

[4] Grundhöfer, T., Automorphism groups of compact projective planes, Geom. Dedicata 21 (1986), 291-298

[5] Hochschild, G., The structure of Lie groups, San Francisco, etc.: Holden-Day 1965

[6] Hofmann, K.H., Strambach, K., Topological and analytical loops, In: O. Chein, H.D. Pflugfelder, J.D.H. Smith (Eds.), Quasigroups and Loops : Theory and Applications, Berlin: Heldermann 1990

[7] Kozma, J., On the differentiability of loopmultiplication in canonical coordinate-system, Publ. Math. Debrecen 37 (1990), 313-325

[8] Kozma, J., Behaviour of loops in a canonical coordinate system, Arch. Math. 55 (1990), $498-502$

[9] Montgomery, D., Zippin, L., Topological transformation groups, New York: Interscience Publ. 1955

[10] Salzmann, H., Homogene kompakte projektive Ebenen, Pac. J. Math. 60 (1975), 217-233

[11] Spivak, M., Differential geometry, Vol. I, Houston: Publish or Perish 1979

[12] Stein, E.M., Singular Integrals and Differentiability Properties of Functions, New Jersey: Princeton University Press 1970

[13] Sternberg, S., Local Contractions and a Theorem of Poincaré, Amer. J. Math. 79 (1957), 809-824

[14] Warner, F.W., Foundations of differentiable manifolds and Lie groups, New York etc.: Springer 1983

Richard Bödi

Linus Kramer

Mathematisches Institut

Universität Tübingen

Auf der Morgenstelle 10

72076 Tübingen

GERMANY mmisa01@mailserv.zdv.uni-tuebingen.de

Eingegangen am 11. November 1993 